\title{
Expression of cyclooxygenase-2 and peroxisome proliferator-activated receptor gamma during malignant melanoma progression
}

Background: Cancer chemoprevention using nonsteroidal antiinflammatory drugs is frequently attributed to cyclooxygenase-2 (COX-2) inhibition, although recent studies suggest that peroxisome proliferator-activated receptor gamma (PPAR $\gamma$ ) may also be involved. While surgical excision remains the treatment mainstay for localized malignant melanoma, certain high-risk patients may benefit from adjunctive chemotherapy. In this study, we compared COX-2 and $\operatorname{PPAR} \gamma$ immunohistological staining in benign nevi, primary melanomas and metastatic melanomas to help predict the effectiveness of compounds targeting these markers.

Methods: COX-2 and PPAR $\gamma$ immunohistological staining was performed and reviewed in 99 melanocytic lesions, including 38 benign nevi, 32 primary melanomas and 29 metastatic melanomas.

Results: There was a significant increase in both COX-2 and PPAR $\gamma$ immunostaining in melanomas compared with benign nevi. Metastatic melanomas were more likely to have a higher number of PPAR $\gamma$ immunopositive cells. They were also more likely to express COX-2 than primary melanomas. Neither COX-2 nor PPAR $\gamma$ expression was associated with a specific pathologic subtype.

Conclusions: COX-2 and PPAR $\gamma$ may help modulate the progression of melanocytic precursor lesions to disseminated malignant melanoma. As such, they may serve as candidate substrates for targeted cancer therapies and may be particularly useful as adjuncts to surgery.

Lee C, Ramirez JA, Guitart J, Diaz LK. Expression of cyclooxygenase-2 and peroxisome proliferator-activated receptor gamma during malignant melanoma progression.

J Cutan Pathol 2008; 35: 989-994. (C) Blackwell Munksgaard 2008.
Carolyn Lee', James A. Ramirez $^{2}$, Joan Guitart ${ }^{3}$ and Leslie K. Diaz ${ }^{3}$

${ }^{1}$ Department of Dermatology, Stanford University School of Medicine, Stanford, CA, USA,

${ }^{2}$ Department of Dermatopathology, St. Joseph Mercy Hospital, Ann Arbor, MI, USA, and ${ }^{3}$ Department of Pathology, Northwestern University School of Medicine, Chicago, IL, USA

Dr Carolyn Lee, Department of Dermatology Stanford University School of Medicine, 900 Blake Wilbur Drive, Room W0069, Stanford, CA 94305, USA

Tel: +16507236316

Fax: 6507237711

e-mail: carilee@stanford.edu

Accepted for publication October 10, 2007
Nonsteroidal anti-inflammatory drugs (NSAIDs) have been shown to be beneficial in the prevention of colorectal and other types of cancer in humans and murine models. ${ }^{1,2}$ This chemopreventive effect is most frequently attributed to cyclooxygenase (COX) inhibition. Expression of the COX-2 isoform has been particularly well studied and is known to be rapidly induced by growth factors and tumor promoters.
Overexpression of COX-2 has been reported in premalignant and malignant lesions derived from a broad spectrum of tissues, including lung, breast, prostate, bladder and the gastrointestinal tract. ${ }^{3}$ Deletion of COX-2 in cancer-prone mice suppresses tumor formation, ${ }^{4}$ and selective COX-2 inhibitors have been shown to provide effective chemoprevention in humans and animal models of 


\section{Lee et al.}

carcinogenesis. ${ }^{5,6}$ These observations suggest that overexpression of COX-2 is an early event in tumorigenesis and may play a role in the progression of precursor lesions to malignant neoplasms. As such, COX-2 may be a suitable candidate molecule for targeted cancer therapies.

Peroxisome proliferator-activated receptor gamma $(\operatorname{PPAR} \gamma)$ is a transcription factor belonging to the nuclear receptor superfamily. ${ }^{7,8}$ It appears to play a role in tumorigenesis, although the exact nature of that role has yet to be defined. A number of studies have shown that PPAR $\gamma$ is expressed in several types of human cancers but not in the normal tissues from which these cancers are derived. ${ }^{9}$ PPAR $\gamma$ agonists have been shown to promote tumorigenesis in cancerprone mice ${ }^{10-12}$ however, the same agonists have also induced differentiation and slowed the growth of human tumor xenografts in other murine models. ${ }^{13-17}$

Studies of PPAR $\gamma$ and COX-2 expression in human skin have yielded mixed results. Immunohistochemical analyses of COX-2 expression in benign nevi and malignant melanomas generally showed an increase in COX-2 expression with cancer progression. ${ }^{18-20}$ Similar studies of PPAR $\gamma$ expression did not detect a correlation with tumor progression but did suggest that an association with COX-2 might exist. $^{21,22}$ Interestingly, PPAR $\gamma$ agonists did not reduce skin tumor formation in two well-characterized models of murine skin carcinogenesis ${ }^{23}$ but did inhibit the growth of human melanoma cell lines in vitro. ${ }^{24,25}$

In an effort to clarify whether PPAR $\gamma$ and COX-2 are suitable molecular markers of melanoma progression, we studied their expression in benign nevi and primary and metastatic melanomas using immunohistochemistry. The data show a progressive increase in COX-2 expression during the malignant progression of melanocytic tumors. Similarly, melanomas, but not benign melanocytic lesions, show increased PPAR $\gamma$ expression. To our knowledge, the data contained herein represent the first immunohistochemistry study to correlate PPAR $\gamma$ expression with progression of malignant melanoma.

\section{Methods}

Histopathological specimens were selected from the files of the Department of Pathology at Northwestern University, Chicago, IL, USA. Each case was independently reviewed by two pathologists (L. K. D and J. A. R), and a consensus was obtained for any discrepant cases.

Immunohistochemical analysis for COX-2 and PPAR $\gamma$ was performed on 38 benign nevi (20 compound, 16 intradermal and 2 junctional), 32 primary melanomas (15 superficial spreading melanomas, 13 nodular melanomas, 2 lentigo maligna melanomas and 2 in situ melanomas) and 29 metastatic melanomas. In six cases, primary and metastatic melanomas were obtained from the same patient. Routine hematoxylin and eosin-stained sections were performed for histopathological evaluation.

Formalin-fixed, paraffin-embedded slides were stained using monoclonal antibodies against PPAR $\gamma$ (dilution 1 : 100; Santa Cruz Biotechnology, Santa Cruz, CA, USA) or COX-2 (catalog number 160112 ; dilution 1 : 100; Cayman Chemical, Ann Arbor, MI, USA) and a standard avidin-biotin-peroxidase complex (ABC) immunoperoxidase procedure. Nuclear staining for PPAR $\gamma$ was classified into four grades on a scale of $0-3$ based on the percentage of positive cells as follows: a score of 0 was given to specimens with $0 \%$ nuclear staining, a score of $1+$ was given to specimens with 1-9\% nuclear staining, a score of $2+$ was given to specimens with 10-50\% nuclear staining and a score of $3+$ was given to specimens with greater than $50 \%$ nuclear staining. COX-2 staining was scored as either weak or strong based on the intensity of cytoplasmic staining.

Fisher's exact test was used to determine statistical significance. The chi-square test was used to determine independence. A p-value of $<0.05$ was considered statistically significant.

\section{Results}

COX-2 expression increases with malignant progression of melanocytic lesions

Virtually all the melanocytic lesions studied showed some level of COX-2 expression. A cytoplasmic pattern of staining was observed (Fig. 1). As a significant number of cases showed a faint background blush, we interpreted COX-2 staining as either strong or weak instead of as a percentage of immunopositive cells. Specimens designated as strong showed intense and crisp cytoplasmic staining. Strong expression of COX-2 was seen in 33\% (33/99) of all lesions studied. The percentage of specimens with strong expression of COX-2 was $3 \%(1 / 38)$ in benign nevi, 38\% (12/32) in primary melanomas and 69\% (20/29) in metastatic melanomas (Fig. 2 and Table 1). Fisher's exact test showed that these differences were statistically significant when comparing benign nevi and primary melanomas $(p=0.0003)$ as well as primary and metastatic melanomas $(\mathrm{p}<0.0001$, Table 2$)$. These data show a progressive increase in COX-2 expression during the malignant progression of melanocytic tumors.

We then compared COX-2 expression in various melanoma subtypes. Thirty-eight percent $(5 / 13)$ of nodular melanomas, $40 \%(6 / 15)$ of superficial spreading melanomas and $50 \%(1 / 2)$ of lentigo maligna melanomas exhibited strong COX-2 expression. There was no statistically significant association 
Fig. 1. Cyclooxygenase-2 expression in representative primary (B) and metastatic (D) melanomas by immunohistochemistry. Hematoxylin and eosin-stained primary and metastatic melanomas are shown in A and C, respectively.
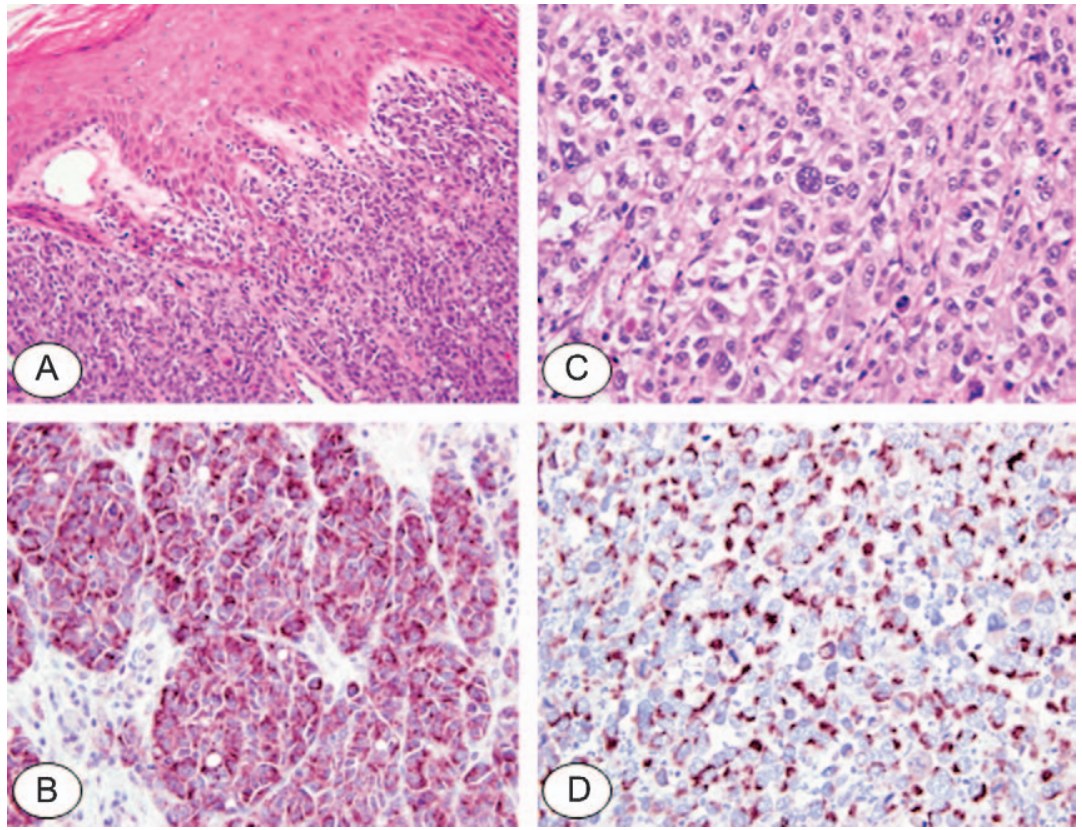

between increased levels of COX-2 and melanoma subtype using Fisher's exact test. Of note, we observed enhanced COX-2 expression in the periphery of two nodular melanomas $(40 \%, 2 / 5)$, consistent with a previous study. ${ }^{19}$

In our study, of the six individuals who had both primary and metastatic melanomas, only two showed strong COX-2 staining of their primary melanomas. These results suggest that strong COX-2 expression in a primary melanoma is not a prognosticator of future metastatic disease, although it is probable that our study was not adequately powered to detect a true difference in this subgroup.

PPAR $\gamma$ is expressed at high levels in primary and metastatic melanomas

Of the 99 samples studied, $22(22 \%)$ showed immunoreactivity against PPAR $\gamma(>10 \%$ immuno-

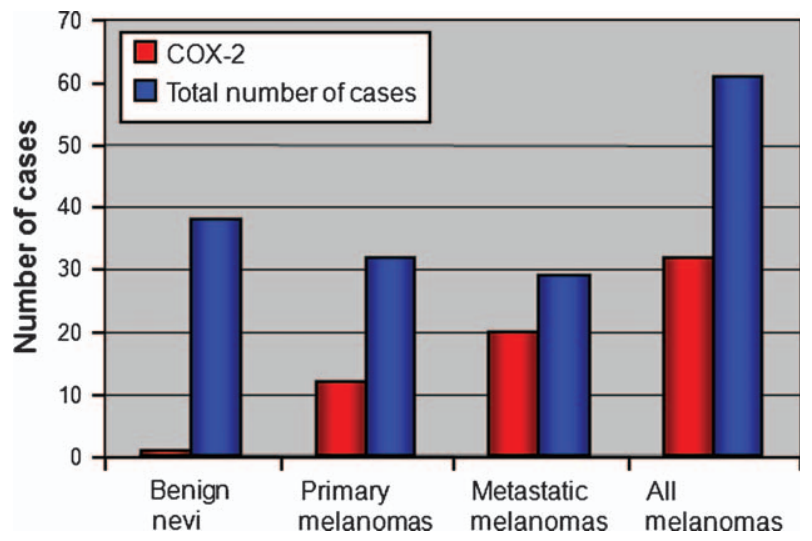

Fig. 2. Frequency of strong cyclooxygenase-2 expression in benign nevi and malignant melanomas. positive keratinocytes). Specifically, 3\% (1/38) benign nevi, 28\% (9/32) primary melanomas and 41\% (12/ 29) metastatic melanomas expressed PPAR $\gamma$ (Table 1). A nuclear staining pattern was predominantly seen (Fig. 3), with cytoplasmic staining of $\operatorname{PPAR} \gamma$ noted in only one metastatic melanoma specimen. Fisher's exact test (Table 2) showed that these differences were statistically significant when comparing benign nevi and primary melanomas $(p=0.004)$ and even more significant when comparing benign nevi and metastatic melanomas $(\mathrm{p}<$ 0.0001). While there appeared to be a trend toward increased PPAR $\gamma$ immunoreactivity in metastatic melanomas compared with primary melanomas, this relationship did not achieve statistical significance $(\mathrm{p}=0.30)$. However, it is important to note that $>50 \%$ nuclear staining was only seen in metastatic melanomas (14\%, 4/29, Table 3). Overall, the data show that increased PPAR $\gamma$ expression occurs in melanomas but not in benign melanocytic lesions.

When we compared PPAR $\gamma$ expression in various melanoma subtypes, 31\% (4/13) of nodular melanomas, $27 \%$ (4/15) of superficial spreading melanomas and $50 \%(1 / 2)$ of lentigo maligna melanomas exhibited PPAR $\gamma$ immunoreactivity. These results

Table 1. COX-2 and PPAR $\gamma$ expression in benign nevi and malignant melanoma

\begin{tabular}{llll}
\hline & $\begin{array}{l}\text { Benign } \\
\text { nevi (\%) }\end{array}$ & $\begin{array}{l}\text { Primary } \\
\text { melanoma (\%) }\end{array}$ & $\begin{array}{l}\text { Metastatic } \\
\text { melanoma (\%) }\end{array}$ \\
\hline COX-2 & $1 / 38(3)$ & $12 / 32(38)$ & $20 / 29(69)$ \\
PPAR $\gamma$ & $1 / 38(3)$ & $9 / 32(28)$ & $12 / 29(41)$ \\
\hline
\end{tabular}

COX-2, cyclooxygenase-2; PPAR $\gamma$, peroxisome proliferator-activated receptor gamma. 


\section{Lee et al.}

Table 2. p-Values of comparisons between melanocytic skin lesions for PPAR $\gamma$ immunoreactivity and strong COX-2 expression

\begin{tabular}{lrc}
\hline Comparisons & \multicolumn{1}{c}{ COX-2* } & PPAR $\gamma^{+}$ \\
\hline Benign nevi vs. primary melanomas & 0.0003 & 0.004 \\
Benign nevi vs. metastatic melanomas & $<0.0001$ & $<0.0001$ \\
Benign nevi vs. all melanomas & $<0.0001$ & $<0.0001$ \\
Primary melanoma vs. metastatic melanomas & $<0.0001$ & 0.30 \\
\hline
\end{tabular}

COX-2, cyclooxygenase-2; PPAR $\gamma$, peroxisome proliferator-activated receptor gamma.

*Intense cytoplasmic immunostaining.

${ }^{+}>10 \%$ immunopositive keratinocytes.

showed no statistically significant association between increased expression of PPAR $\gamma$ and melanoma subtype using Fisher's exact test.

In our study, of the six individuals who had both primary and metastatic melanomas, only two had primary melanomas that expressed $\operatorname{PPAR} \gamma$. These results suggest that PPAR $\gamma$ expression in a primary melanoma is not a prognosticator of future metastatic disease, although we cannot rule it out completely because of our limited sample size.

\section{Discussion}

Identifying molecular markers of cancer progression is critical for sustaining the current focus on targeted cancer therapies and rational drug design. An early intervention capable of interrupting or reversing disease progression would be particularly useful in malignant melanoma, where the current standards of care for metastatic disease have not resulted in a significant survival benefit. Targeted therapies could also be used as an adjunct to surgical excision in patients with localized disease who are at high risk of developing future metastases. To our knowledge, this is the first study to report increased expression of PPAR $\gamma$ in human malignant melanoma. This finding may explain why melanoma cell lines appear susceptible to the antiproliferative effects of selective PPAR $\gamma$ ligands, such as thiazolidinediones. ${ }^{21,25}$

In this study, we show that PPAR $\gamma$ is expressed in a significant proportion of primary and metastatic melanomas but rarely expressed in benign nevi. Furthermore, $>50 \%$ nuclear staining was only seen in metastatic melanomas, suggesting that PPAR $\gamma$ may have a role in the modulation of tumor invasion. We considered the possibility that strong expression of PPAR $\gamma$ in a primary melanoma might predict future metastases. However, our study was not adequately powered to perform this analysis. It is nonetheless interesting to note that thiazolidinediones were recently shown to inhibit cell migration and invasion in human breast and pancreatic cell lines. ${ }^{26,27}$ Our data suggest that similar results may be seen in melanoma as well.

There are differing reports on the cellular localization of PPAR $\gamma$ in the skin. Some studies have observed a granular cytoplasmic pattern, while others have noted nuclear staining. ${ }^{21,22}$ Our results are consistent with those obtained by others who used the same PPAR $\gamma$ antibody, suggesting that reagent selection may influence apparent localization. It is also possible that the discrepancy between the data described in Nijsten et al. ${ }^{22}$ and those described herein is because of tumor-specific differences in $\operatorname{PPAR} \gamma$ localization.

Increased expression of PPAR $\gamma$ has been reported in a wide range of human cancers. In melanoma cell lines, PPAR $\gamma$ agonists appear to suppress cellular
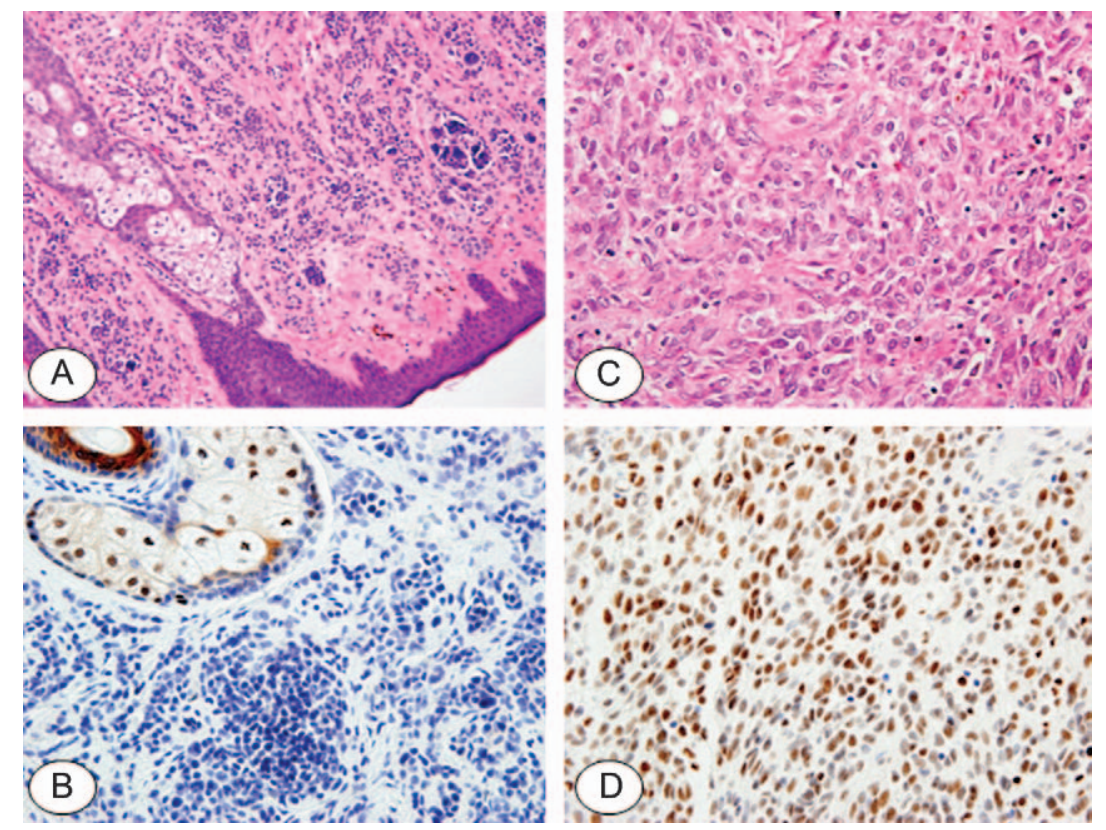

Fig. 3. Peroxisome proliferator-activated receptor gamma expression in a representative benign nevus (B) and metastatic melanoma (D) by immunohistochemistry. Of note, the sebaceous gland in B serves as an internal positive control. A hematoxylin and eosinstained benign nevus and a metastatic melanoma are shown in $\mathrm{A}$ and $\mathrm{C}$, respectively. 
Table 3. Peroxisome proliferator-activated receptor gamma immunoreactivity in melanocytic skin lesions

\begin{tabular}{llll}
\hline & $\begin{array}{l}0-10 \% \\
\text { nuclear } \\
\text { staining (\%) }\end{array}$ & $\begin{array}{l}10-50 \% \\
\text { nuclear } \\
\text { staining (\%) }\end{array}$ & $\begin{array}{l}>50 \% \\
\text { nuclear } \\
\text { staining (\%) }\end{array}$ \\
\hline Benign nevi & $37 / 38(97)$ & $1 / 38(3)$ & $0 / 38(0)$ \\
Primary melanomas & $23 / 32(72)$ & $9 / 32(28)$ & $0 / 32(0)$ \\
Metastatic melanomas & $17 / 29(59)$ & $8 / 29(27)$ & $4 / 29(14)$ \\
\hline
\end{tabular}

proliferation and induce differentiation. ${ }^{21,24,25,28}$ However, inhibition of PPAR $\gamma$ has produced similar results in other cancer types, including oral squamous cell carcinomas and hepatocellular carcinomas. ${ }^{29,30}$ We are not certain why this discrepancy occurs, but it may be the result of off-target effects of PPAR $\gamma$ ligands or inherent differences between cell lines.

Strong COX-2 expression was observed in both primary and metastatic melanomas but rarely in benign nevi. Of note, we found that metastatic melanomas were significantly more likely to have strong COX-2 expression than primary melanomas. Our results are consistent with previous studies, ${ }^{19,20}$ although we further extend the findings of Kuzbicki et al. to include distant cutaneous metastases as well as lymph node metastases. The data also raise the possibility that COX-2 promotes melanoma cell invasiveness. Our preliminary analysis did not detect a correlation between strong COX-2 expression in a primary melanoma and the presence of metastatic disease, but we recognize that the small sample size makes it difficult to rule out a type II error. Pharmacologic inhibition of COX-2 in melanoma cell lines has not consistently reduced prostaglandin E2 production or tumor cell invasiveness. ${ }^{19,31}$ It is interesting to speculate whether this is related to offtarget effects of NSAIDs, as a COX-independent mechanism of tumor suppression by these compounds has been shown. ${ }^{32-34}$ Additional studies to clarify the role of COX-2 in melanoma progression and invasion seem to be warranted.

Studies on colorectal cancer have suggested that COX-2 and PPAR $\gamma$ may have opposing effects on tumorigenesis. ${ }^{35}$ More recently, an immunohistochemical study showed that COX-2-positive squamous cell carcinomas and actinic keratoses were more likely to express PPAR $\gamma$ or its isoform, PPAR $\beta .^{22}$ As such, we expected to see a correlation between COX2 and PPAR $\gamma$ expression in our study. A chi-square test for independence surprisingly did not show a relationship between COX-2 and PPAR $\gamma$. While we cannot say for certain why this discrepancy occurred, it is possible that cancer-specific differences may be involved.

Our study examines the expression patterns of PPAR $\gamma$ and COX-2 in melanocytic lesions of the skin. The data suggest that both proteins play a role in the development and biological behavior of melanomas.
More importantly, increased expression of these markers in melanoma may enable us to tailor treatment to selectively target malignant cells. The prothrombotic and gastrointestinal complications of COX-2 inhibitors have raised concerns about their long-term safety, although these risks may be outweighed by a potential benefit in survival in high-risk, disseminated or unresectable disease. Our data showing increased PPAR $\gamma$ expression in primary and metastatic melanomas also provide an explanation for the observed efficacy of thiazolidinediones in melanoma cell lines. As such, drugs that target COX-2 or PPAR $\gamma$, particularly those with favorable safety profiles, may be effective adjuncts in the treatment of melanoma.

\section{References}

1. Waddell WR, Loughry RW. Sulindac for polyposis of the colon. J Surg Oncol 1983; 24: 83.

2. Smalley WE, DuBois RN. Colorectal cancer and nonsteroidal anti-inflammatory drugs. Adv Pharmacol 1997; 39: 1.

3. Dannenberg AJ, Altorki NK, Boyle JO, et al. Cyclo-oxygenase 2: a pharmacological target for the prevention of cancer. Lancet Oncol 2001; 2: 544.

4. Oshima M, Dinchuk JE, Kargman SL, et al. Suppression of intestinal polyposis in Apc delta716 knockout mice by inhibition of cyclooxygenase 2 (COX-2). Cell 1996; 87: 803.

5. Steinbach G, Lynch PM, Phillips RK, et al. The effect of celecoxib, a cyclooxygenase-2 inhibitor, in familial adenomatous polyposis. N Engl J Med 2000; 342: 1946.

6. Kawamori T, Rao GV, Seibert K, Reddy BS. Chemopreventive activity of celecoxib, a specific cyclooxygenase-2 inhibitor, against colon carcinogenesis. Cancer Res 1998; 58: 409.

7. Lehmann JM, Lenhard JM, Oliver BB, Ringold GM, Kliewer SA. Peroxisome proliferator-activated receptors alpha and gamma are activated by indomethacin and other non-steroidal anti-inflammatory drugs. J Biol Chem 1997; 272: 3406.

8. Yamazaki R, Kusunoki N, Matsuzaki T, Hashimoto S, Kawai S. Nonsteroidal anti-inflammatory drugs induce apoptosis in association with activation of peroxisome proliferator-activated receptor gamma in rheumatoid synovial cells. J Pharmacol Exp Ther 2002; 302: 18.

9. Koeffler HP. Peroxisome proliferator-activated receptor gamma and cancers. Clin Cancer Res 2003; 9: 1.

10. Lefebvre AM, Chen I, Desreumaux P, et al. Activation of the peroxisome proliferator-activated receptor gamma promotes the development of colon tumors in C57BL/6J-APCMin/+ mice. Nat Med 1998; 4: 1053.

11. Saez E, Tontonoz P, Nelson MC, et al. Activators of the nuclear receptor PPARgamma enhance colon polyp formation. Nat Med 1998; 4: 1058.

12. Saez E, Rosenfeld J, Livolsi A, et al. PPAR gamma signaling exacerbates mammary gland tumor development. Genes Dev 2004; 18: 528.

13. Sarraf P, Mueller E, Jones D, et al. Differentiation and reversal of malignant changes in colon cancer through PPARgamma. Nat Med 1998; 4: 1046.

14. Mueller E, Sarraf P, Tontonoz P, et al. Terminal differentiation of human breast cancer through PPAR gamma. Mol Cell 1998; 1: 465 . 


\section{Lee et al.}

15. Elstner E, Muller C, Koshizuka K, et al. Ligands for peroxisome proliferator-activated receptorgamma and retinoic acid receptor inhibit growth and induce apoptosis of human breast cancer cells in vitro and in BNX mice. Proc Natl Acad Sci U S A 1998; 95: 8806.

16. Kubota T, Koshizuka K, Williamson EA, et al. Ligand for peroxisome proliferator-activated receptor gamma (troglitazone) has potent antitumor effect against human prostate cancer both in vitro and in vivo. Cancer Res 1998; 58: 3344.

17. Tontonoz P, Singer S, Forman BM, et al. Terminal differentiation of human liposarcoma cells induced by ligands for peroxisome proliferator-activated receptor gamma and the retinoid X receptor. Proc Natl Acad Sci U S A 1997; 94: 237.

18. Goulet AG, Einsphar JG, Alberts DS, et al. Analysis of cyclooxygenase 2 (COX-2) expression during malignant melanoma progression. Cancer Biol Ther 2003; 2: 713.

19. Denkert C, Kobel M, Berger S, et al. Expression of cyclooxygenase 2 in human malignant melanoma. Cancer Res 2001; 61: 303.

20. Kuzbicki L, Sarnecka A, Chwirot BW. Expression of cyclooxygenase-2 in benign naevi and during human cutaneous melanoma progression. Melanoma Res 2006; 16: 29.

21. Mossner R, Schulz U, Kruger U, et al. Agonists of peroxisome proliferator-activated receptor gamma inhibit cell growth in malignant melanoma. J Invest Dermatol 2002; 119: 576.

22. Nijsten T, Geluyckens E, Colpaert C, Lambert J. Peroxisome proliferator-activated receptors in squamous cell carcinoma and its precursors. J Cutan Pathol 2005; 32: 340.

23. He G, Muga S, Thuillier P, Lubet RA, Fischer SM. The effect of PPARgamma ligands on UV- or chemically-induced carcinogenesis in mouse skin. Mol Carcinog 2005; 43: 198.

24. Placha W, Gil D, Dembinska-Kiec A, Laidler P. The effect of PPARgamma ligands on the proliferation and apoptosis of human melanoma cells. Melanoma Res 2003; 13: 447.

25. Freudlsperger C, Moll I, Schumacher U, Thies A. Antiproliferative effect of peroxisome proliferator-activated receptor gamma agonists on human malignant melanoma cells in vitro. Anticancer Drugs 2006; 17: 325.
26. Liu H, Zang C, Fenner MH, Possinger K, Elstner E. PPARgamma ligands and ATRA inhibit the invasion of human breast cancer cells in vitro. Breast Cancer Res Treat 2003; 79: 63.

27. Motomura W, Nagamine M, Tanno S, et al. Inhibition of cell invasion and morphological change by troglitazone in human pancreatic cancer cells. J Gastroenterol 2004; 39: 461.

28. Liu Y, Meng Y, Liu H, et al. Growth inhibition and differentiation induced by peroxisome proliferator activated receptor gamma ligand rosiglitazone in human melanoma cell line a375. Med Oncol 2006; 23: 393.

29. Masuda T, Wada K, Nakajima A, et al. Critical role of peroxisome proliferator-activated receptor gamma on anoikis and invasion of squamous cell carcinoma. Clin Cancer Res 2005; 11: 4012.

30. Schaefer KL, Wada K, Takahashi H, et al. Peroxisome proliferator-activated receptor gamma inhibition prevents adhesion to the extracellular matrix and induces anoikis in hepatocellular carcinoma cells. Cancer Res 2005; 65: 2251.

31. Denkins Y, Kempf D, Ferniz M, Nileshwar S, Marchetti D. Role of omega-3 polyunsaturated fatty acids on cyclooxygenase-2 metabolism in brain-metastatic melanoma. J Lipid Res 2005; 46: 1278.

32. Zhang X, Morham SG, Langenbach R, Young DA. Malignant transformation and antineoplastic actions of nonsteroidal antiinflammatory drugs (NSAIDs) on cyclooxygenase-null embryo fibroblasts. J Exp Med 1999; 190: 451.

33. Sawaoka H, Tsuji S, Tsujii M, et al. Cyclooxygenase inhibitors suppress angiogenesis and reduce tumor growth in vivo. Lab Invest 1999; 79: 1469.

34. Wechter WJ, Kantoci D, Murray ED Jr, et al. R-flurbiprofen chemoprevention and treatment of intestinal adenomas in the $\mathrm{APC}(\mathrm{Min}) /+$ mouse model: implications for prophylaxis and treatment of colon cancer. Cancer Res 1997; 57: 4316.

35. Park BH, Breyer B, He TC. Peroxisome proliferator-activated receptors: roles in tumorigenesis and chemoprevention in human cancer. Curr Opin Oncol 2001; 13: 78. 